\title{
Phase String Effect in a Doped Antiferromagnet
}

\author{
D. N. Sheng, Y.C. Chen,* and Z. Y. Weng \\ Texas Center for Superconductivity and Department of Physics, University of Houston, Houston, Texas 77204-5506
}

(Received 27 June 1996)

\begin{abstract}
Based on the $t-J$ model, it is shown that a hole moving on an antiferromagnetic spin background always induces a phase-string effect. Such a previously unnoticed phase string is revealed by explicitly tracking the Marshall sign and can be rigorously shown to be nonrepairable at low energy. Its quantum interference can drastically modify the long-wavelength behavior of the doped hole, leading to a vanishing spectral weight $Z$ at the ground-state energy. Implication for finite doping is also discussed. [S0031-9007(96)01864-9]
\end{abstract}

PACS numbers: 71.27.+a, 74.20.Mn, 75.10.Jm

A hole injected into a two-dimensional (2D) antiferromagnet has been studied extensively [1-3] based on the $t$ - $J$ model. It is well known that a string of spin mismatches left by the hopping of the injected hole on the spin background can be repaired through the spin flip process. Consequently the hole is believed to be mobile without a confining potential linearly proportional to the length of the path. However, whether such a mobile hole can be described as a quasiparticle is still controversial.

The key issue [4] involves the spectral weight $Z$ at the ground-state energy $E_{G} . Z\left(E_{G}\right) \neq 0$ means a finite overlap of the "bare" hole state with the true ground state. It implies that the real hole in the ground state behaves like a spin polaron with the bare hole carrying a spin distortion around it. If $Z\left(E_{G}\right)=0$, then each injected hole will cause a global adjustment in the original ground state with a breakdown of the perturbatively treatable quasiparticle picture. The main difficulty in this problem arises from the fact that as different from the usual phonon-polaron picture, $S U(2)$ spins are involved here and a U(1) phase plays an important role in shaping the long-distance part of a spin polaron with little energy cost. The spectral weight $Z\left(E_{G}\right)$ at the ground-state energy is particularly sensitive to such long-wavelength, lowenergy correlations in the system. Even though exact diagonalization calculations on small lattices $[2,3]$ have indicated a quasiparticle peak at the energy bottom of the spectral function, when the lattice size goes to infinity it is hard to tell whether such a quasiparticle peak would still stay at the lower end of the spectra or there could be some weight (e.g., a tail) emerging below the peak which vanishes at the ground-state energy [such that $\left.Z\left(E_{G}\right)=0\right]$. Therefore, a more accurate approach would be desirable in order to get access to this long-distance regime. In this Letter, by explicitly tracking the Marshall sign, we show that the injected hole always has to pick up a sequence of signs (phases) from the quantum spin background. In contrast to the well-known repairable string defect of spin mismatches, we find that the phase string revealed here cannot be dynamically "eliminated" through the low-lying spin dynamics. Consequently, it dramatically modifies the long-wavelength behaviors of the doped hole. In particular, the spectral weight $Z\left(E_{G}\right)$ has to vanish due to such a nonrepairable phase-string effect.

Let us start with the undoped case. It is described by the superexchange Hamiltonian

$$
H_{J}=J \sum_{\langle i j\rangle}\left[\mathbf{S}_{i} \cdot \mathbf{S}_{j}-\frac{n_{i} n_{j}}{4}\right],
$$

which is equivalent to the Heisenberg model as the electron occupation number $n_{i}=n_{j}=1$. According to Marshall [5], the ground-state wave function of the Heisenberg Hamiltonian for a bipartite lattice is real and satisfies a sign rule. This sign rule requires that any flips of two antiparallel spins at nearest-neighboring sites are always accompanied by a sign change in the wave function: i.e., $\uparrow \downarrow \rightarrow(-1) \downarrow \uparrow$. Such a Marshall sign rule may be easily understood as below. Suppose that one has a complete set of spin bases $\{|\phi\rangle\}$ with the built-in Marshall sign. It is straightforward to verify that matrix elements of $H_{J}$ become negative definite: $\left\langle\phi^{\prime}\left|H_{J}\right| \phi\right\rangle \leq 0$. Then, for the ground state $\left|\psi_{0}\right\rangle=\sum_{\phi} \chi_{\phi}|\phi\rangle$ one finds that the coefficient $\chi_{\phi}$ should be always real and positive (except for a trivial global phase) in order to reach the lowest energy. It means that the Marshall sign is indeed the only sign showing up in the ground state.

There can be many ways to incorporate the Marshall sign into the $S^{z}$-spin representation. We may divide a bipartite lattice into even $(A)$ and odd $(B)$ sublattices and assign an extra sign -1 to each down spin at $A$ site. In this way, flips of two nearest-neighboring antiparallel spins always involve a down spin changing sublattices, and thus a sign change. Of course, this is not a unique way to incorporate the Marshall sign in the spin basis, but it will be quite useful bookkeeping once a hole is introduced into the system. Such a spin basis with one hole can be defined as

$$
|\phi ;(n)\rangle=(-1)^{N_{A}^{\downarrow}}|\uparrow \ldots \downarrow \uparrow \circ \ldots \downarrow\rangle,
$$


with $n$ denoting the hole site and $N_{A}^{\downarrow}$ as the total number of down spins at $A$ sublattice. It is easy to check that

$$
\left\langle\phi^{\prime} ;(n)\left|H_{J}\right| \phi ;(n)\right\rangle \leq 0 .
$$

It means that the Marshall sign rule would be still satisfied if the hole is fixed at a given site $n$.

Now we consider the hopping of the hole. The hopping process is governed by $H_{t}$ in the $t-J$ model which is defined by

$$
H_{t}=-t \sum_{\langle i j\rangle} c_{i \sigma}^{\dagger} c_{j \sigma}+\text { H.c. }
$$

where the Hilbert space is restricted by the no-doubleoccupancy constraint $\sum_{\sigma} c_{i \sigma}^{\dagger} c_{i \sigma} \leq 1$. Suppose that the hole initially sitting at site $n$ hops onto a nearestneighboring site $m$. The corresponding matrix element under the basis (2) can be easily found to be

$$
\left\langle\phi^{\prime} ;(m)\left|H_{t}\right| \phi ;(n)\right\rangle=-t \times \sigma_{m},
$$

where $\sigma_{m}$ is the site- $m$ spin index in the state $|\phi ;(n)\rangle$ and $\phi^{\prime}$ is different from $\phi$ by an exchanging of the spin $\sigma_{m}$ with the hole at site $n$. Since $\sigma_{m}= \pm 1$, the hopping matrix element is not sign definite. In other words, the hopping process will lead to the violation of the Marshall sign rule in the ground state. In the following, we shall explore this phase "frustration" effect in detail.

We define a bare hole to be described by $c_{i \sigma}\left|\psi_{0}\right\rangle$. One can track its evolution by studying the propagator $G_{1 \sigma}(j, i ; E)=\left\langle\psi_{0}\left|c_{j \sigma}^{\dagger}\left(E-H_{t-J}+i \eta\right)^{-1} c_{i \sigma}\right| \psi_{0}\right\rangle$, with $H_{t-J}=H_{t}+H_{J}$ and $\eta=0^{+}$. To separate the hopping and superexchange processes, one may expand $G_{1 \sigma}$ in terms of $H_{t}$ as follows:

$$
G_{1 \sigma}(j, i ; E)=\left\langle\psi_{0}\left|c_{j \sigma}^{\dagger}\left(G_{J}+G_{J} H_{t} G_{J}+\ldots\right) c_{i \sigma}\right| \psi_{0}\right\rangle,
$$

where $G_{J} \equiv\left(E-H_{J}+i \eta\right)^{-1}$. The bare hole state can be written as $c_{i \sigma}\left|\psi_{0}\right\rangle=(\sigma)^{i} \sum_{\phi} \chi_{\phi(i \sigma)}|\phi ;(i)\rangle$ where $\phi(i \sigma)$ refers to any possible spin configurations with a fixed spin $\sigma$ at site $i$, and if $\sigma=-1,(\sigma)^{i}=(-1)^{i}=$ -1 at $A$-sublattice site and +1 at $B$-sublattice site. Then, by inserting the complete set of the basis (2) as intermediate states, one obtains

$$
\begin{aligned}
G_{1 \sigma}(j, i ; E)= & (\sigma)^{j-i} \sum_{\text {(all paths) (all states) }} \chi_{\phi^{\prime}(j \sigma)} \chi_{\phi(i \sigma)} \\
& \times T_{i j}(\{\phi\}) \prod_{s=0}^{K_{i j}}\left\langle\phi^{s+1} ;\left(m_{s}\right)\left|G_{J}(E)\right| \phi^{s} ;\left(m_{s}\right)\right\rangle,
\end{aligned}
$$

where $\left|\phi^{s} ;\left(m_{s}\right)\right\rangle$ and $\left|\phi^{s+1} ;\left(m_{s}\right)\right\rangle$ describe two different spin configurations $\left\{\phi^{s}\right\}$ and $\left\{\phi^{s+1}\right\}$ with the hole sitting at site $m_{s}$ on a given path connecting sites $i$ and $j: m_{0}=$ $i, m_{1}, \ldots, m_{K_{i j}}=j$ [here $K_{i j}$ is the total number of links for the given path, and $\left.\phi^{0} \equiv \phi(i \sigma), \phi^{K_{i j}+1} \equiv \phi^{\prime}(j \sigma)\right]$.
$T_{i j}$ is a product of matrices of $H_{t}$ which connects $\left\{\left|\phi_{\left(m_{s}\right)}^{s+1}\right\rangle\right\}$ with $\left\{\left|\phi_{\left(m_{s+1}\right)}^{s+1}\right\rangle\right\}$ for such a path:

$$
T_{i j}(\{\phi\})=\prod_{k=1}^{K_{i j}}(-t) \sigma_{m_{k}},
$$

where $\sigma_{m_{k}}$ denotes the instant spin state at site $m_{k}$ right before the hole hops to it.

We may further write $G_{1 \sigma}(j, i ; E)$ in a more compact form, namely,

$$
\begin{aligned}
G_{1 \sigma}(j, i ; E)= & -(\sigma)^{j-i} \sum_{\text {(all paths) }} \\
& \times \sum_{\{\phi\}} W_{\text {path }}[\{\phi\}]\left(\prod_{k=1}^{K_{i j}} \sigma_{m_{k}}\right),
\end{aligned}
$$

where the summation over $\{\phi\}$ means summing over all the possible spin configurations in the initial and final as well as intermediate states. Here $W_{\text {path }}[\{\phi\}]$ is defined by

$$
\begin{aligned}
W_{\text {path }}[\{\phi\}]= & \frac{1}{t} \chi_{\phi^{\prime}(j \sigma)} \chi_{\phi(i \sigma)} \prod_{s=0}^{K_{i j}}(-t) \\
& \times\left\langle\phi^{s+1} ;\left(m_{s}\right)\left|G_{J}(E)\right| \phi^{s} ;\left(m_{s}\right)\right\rangle .
\end{aligned}
$$

In the following, we prove that $W_{\text {path }}[\{\phi\}]$ is always positive definite near the ground-state energy. To determine the sign of $\left\langle\phi^{s+1} ;\left(m_{s}\right)\left|G_{J}(E)\right| \phi^{s} ;\left(m_{s}\right)\right\rangle$, one may expand $G_{J}$ as follows:

$$
G_{J}(E)=\frac{1}{E} \sum_{k} \frac{H_{J}^{k}}{E^{k}} .
$$

Note that $\left\langle\phi^{s+1} ;\left(m_{s}\right)\left|H_{J}^{k}\right| \phi^{s} ;\left(m_{s}\right)\right\rangle=(-1)^{k} \mid\left\langle\phi^{s+1}\right.$; $\left.\left(m_{s}\right)\left|H_{J}^{k}\right| \phi^{s} ;\left(m_{s}\right)\right\rangle \mid$ [one may easily show it by writing $H_{J}^{k}=H_{J} \cdot H_{J} \cdot \ldots$ and inserting the complete set of (2) in between and using the condition (3)]. Then one finds

$$
\begin{aligned}
& \left\langle\phi^{s+1} ;\left(m_{s}\right)\left|G_{J}(E)\right| \phi^{s} ;\left(m_{s}\right)\right\rangle \\
& =\frac{1}{E} \sum_{k} \frac{\left|\left\langle\phi^{s+1}\left(m_{s}\right)\left|H_{J}^{k}\right| \phi^{s+1} ;\left(m_{s}\right)\right\rangle\right|}{(-E)^{k}}<0,
\end{aligned}
$$

if $E<0$. Of course one still needs to determine the convergence range of the expansion. By inserting a complete set of eigenstates as intermediate states, it is easy to see that $\left\langle\phi^{s+1} ;\left(m_{s}\right)\left|G_{J}(E)\right| \phi^{s} ;\left(m_{s}\right)\right\rangle$ is an analytic function of $E$ except for a branch cut on the real axis covered by the eigenvalues of $H_{J}$ (with a hole fixed at site $m_{s}$ ). This analytic property will guarantee the convergence of the expansion (12) in the whole region of $E<E_{G}^{0}<0$ on the real axis, where $E_{G}^{0}$ is the lowest energy of the eigenvalues of $H_{J}$ with the hole fixed on a lattice site. We note that $E_{G}^{0}$ is always higher than the true ground-state energy $E_{G}$ of $H_{t-J}$, where the hole is allowed to move around to gain its kinetic energy. Thus, near the ground-state energy $E_{G}$, one always has $W_{\text {path }}[\{\phi\}] \geq 0$. 
Therefore, we see in (9) that the hole has picked up a sequence of signs $\prod_{k=1}^{K_{i j}} \sigma_{m_{k}}=( \pm 1) \times( \pm 1) \times$ $\ldots \times( \pm 1)$ from the spin background. A sign-definite $W_{\text {path }}[\{\phi\}]$ in the propagator (9) means that the spin background cannot produce any phases to "compensate" such a phase string. This subtle phase-string effect has been overlooked before, but is expected to play a crucial role in determining the long-wavelength behavior of the hole, due to its nonrepairable nature.

Since $W_{\text {path }}[\{\phi\}]$ is sign definite, one may introduce the following weight functional: $\rho_{\text {path }}[\{\phi\}]=$ $W_{\text {path }}[\{\phi\}] / \sum_{\text {(all paths) }} \sum_{\{\phi\}} W_{\text {path }}[\{\phi\}]$, which satisfies the condition $\sum_{\text {(all paths) }} \sum_{\{\phi\}} \rho_{\text {path }}[\{\phi\}]=1$. Then the propagator $G_{1 \sigma}$ can be expressed in the following form:

$$
G_{1 \sigma}(j, i ; E)=\tilde{G}_{1 \sigma}(j, i ; E)\left\langle\prod_{k=1}^{K_{i j}} \sigma_{m_{k}}\right\rangle,
$$

where

$$
\tilde{G}_{1 \sigma}(j, i ; E) \equiv-(\sigma)^{j-i} \sum_{\text {(all paths) }\{\phi\}} \sum_{\text {path }}[\{\phi\}],
$$

and

$$
\left\langle\prod_{k=1}^{K_{i j}} \sigma_{m_{k}}\right\rangle \equiv \sum_{\text {(all paths) }} \sum_{\{\phi\}} \rho_{\text {path }}[\{\phi\}]\left(\prod_{k=1}^{K_{i j}} \sigma_{m_{k}}\right)
$$

$\tilde{G}_{1 \sigma}(j, i ; E)$ defined in (14) may be regarded as the singlehole propagator under a new Hamiltonian $\tilde{H}_{t-J}$ obtained by replacing the hopping term $H_{t}$ in the $t-J$ model with $\tilde{H}_{t}$, whose matrix element is negative definite $(-t)$ without the extra sign problem shown in (5).

Thus the nonrepairable signs picked up by the hole are sorted out in (13) and the real hole propagator is modulated by a superposition of phase strings from different paths under all possible spin configurations. In the following we give a general argument that $\left\langle\prod_{k=1}^{K_{i j}} \sigma_{m_{k}}\right\rangle$ has to vanish at $|i-j| \rightarrow \infty$.

We may reexpress the phase-string factor for a given path as follows:

$$
\prod_{k=1}^{K_{i j}} \sigma_{m_{k}}=(-1)^{N_{\text {path }}^{\downarrow},}
$$

where $N_{\text {path }}^{\downarrow}$ denotes the total number of $\downarrow$ spins “exchanged" with the hole as it moves from $i$ to $j$. Notice that $(-1)^{N_{\text {path }}^{\downarrow}+N_{\text {path }}^{\dagger}} \equiv(-1)^{j-i}$ which is independent of the path and thus the system is symmetric about $\uparrow$ and $\downarrow$ spins. Such a phase-string factor defined in (16) is quite singular as it changes a sign each time when the total number of $N_{\text {path }}^{\downarrow}$ increases or decreases by one, no matter how long the path is. Therefore, its average crucially depends on the total number fluctuating of $\Delta N_{\text {path }}^{\downarrow}=-\Delta N_{\text {path }}^{\uparrow}$ $\left(\Delta N_{\text {path }}^{\sigma} \equiv N_{\text {path }}^{\sigma}-\bar{N}_{\text {path }}^{\sigma}\right)$. Here the number fluctuating is due to different spin configurations encountered by the hole (each spin flip gives rise to $\Delta N^{\downarrow}= \pm 1$ ). Even in the presence of the long-range spin order in the thermo- dynamic limit zero-point fluctuations are always present which means that the hole still gets to see a large fluctuation of $N_{\text {path }}^{\downarrow}$ along a sufficiently long path.

One may distinguish two cases regarding the fluctuation of $\Delta N_{\text {path }}^{\downarrow}$ at the limit of $|i-j| \rightarrow \infty$. One is that $\left\langle\left|\Delta N_{\text {path }}^{\downarrow}\right|\right\rangle \rightarrow \infty$, and the other is that $\left\langle\left|\Delta N_{\text {path }}^{\downarrow}\right|\right\rangle \sim O(1)$. Generally one expects to see $\left\langle\left|\Delta N_{\text {path }}^{\downarrow}\right|\right\rangle \rightarrow \infty$ as the result that the "randomness" of spin flips on the path is accumulated in this limit. On the other hand, one could not rule out the possibility that $\left\langle\left|\Delta N_{\text {path }}^{\downarrow}\right|\right\rangle \sim O(1)$ if there exists a hidden symmetry in the system which guarantees that $\Delta N_{\text {path }}^{\downarrow}$ does not grow up with the distance of the path, namely, if $\uparrow$ and $\downarrow$ spins are paired up at short distance within the same path. In this way, the fluctuations due to flips of those spin pairs will not change $\Delta N_{\text {path }}^{\downarrow}$, since a down flip of an $\uparrow$ spin is always accompanied by an up flip of a $\downarrow$ spin on the same path, even though the number of those "flips" can still increase indefinitely at $|i-j| \rightarrow \infty$. Then the total fluctuating number $\Delta N_{\text {path }}^{\downarrow}$ could maintain predominantly to be finite even in an infinite-long path. In this case, $\left\langle(-1)^{N_{\text {path }}^{\downarrow}}\right\rangle$ can remain finite: e.g., $\left\langle(-1)^{N_{\text {path }}^{\downarrow}}\right\rangle=$ $\operatorname{Re}\left\langle e^{i \pi N_{\mathrm{path}}^{\downarrow}}\right\rangle \approx \operatorname{Re}\left(e^{i \pi\left\langle N_{\mathrm{path}}^{\downarrow}\right\rangle}\right) e^{-\frac{\pi^{2}}{2}\left\langle\left(\Delta N_{\mathrm{path}}\right)^{2}\right\rangle} \sim$ finite at $|i-j| \rightarrow \infty$. This could happen in a one-dimensional case with anisotropic superexchange spin coupling [6], where there is only one nonrepeatable path connecting $i$ and $j$ and, at the same time, $\uparrow$ and $\downarrow$ spins are all short-ranged paired. In the $1 \mathrm{D}$ isotropic case, the spin excitation gap vanishes and the spin(on) pairs become quasilong ranged (power-law decay) [7] such that one still finds $\left\langle\left|\Delta N_{\text {path }}^{\downarrow}\right|\right\rangle \rightarrow \infty$ at $|i-j| \rightarrow \infty$. At two or higher dimensions, even if spins are short-ranged paired, each pair cannot always be present simultaneously within the same path. Thus one generally finds that the average fluctuating of $N_{\text {path }}^{\downarrow}$ relative to $\bar{N}_{\text {path }}^{\downarrow}$ increases indefinitely at long distances. As $\left\langle\left|\Delta N_{\text {path }}^{\downarrow}\right|\right\rangle \rightarrow \infty$, the probabilities for a $N_{\text {path }}^{\downarrow}$ and $N_{\text {path }}^{\downarrow} \rightarrow N_{\text {path }}^{\downarrow} \pm 1$ become indistinguishable in this "thermodynamic" limit. Consequently, the average $\left\langle(-1)^{N_{\text {path }}^{\downarrow}}\right\rangle$ has to vanish at $|i-j| \rightarrow \infty$.

Therefore, in a general case $G_{1 \sigma}(j, i ; E)$ will always decay, at least, in the speed that $\left\langle\prod_{k=1}^{K_{i j}} \sigma_{m_{k}}\right\rangle$ decays at large distance (note that $\tilde{G}_{1 \sigma}(j, i ; E)$ as a propagator may either decay to zero or become stationary at $|i-j| \rightarrow$ $\infty)$. In particular, $G_{1 \sigma}(j, i ; E)$ decays at the ground-state energy $E=E_{G}$. It means $Z(E)=0$ at $E \rightarrow E_{G}$. To see it, let us consider the imaginary part of $G_{1 \sigma}(k, E)$ in momentum space, which is given by

$$
\operatorname{Im} G_{1 \sigma}(k, E)=-\pi \sum_{M} Z_{k}\left(E_{M}\right) \delta\left(E-E_{M}\right),
$$

where the spectral weight $Z_{k}$ is defined as

$$
Z_{k}\left(E_{M}\right)=\left|\left\langle\psi_{M}\left|c_{k \sigma}\right| \psi_{0}\right\rangle\right|^{2},
$$

with $\left|\psi_{M}\right\rangle$ and $E_{M}$ denoting the eigenstate and energy of $H_{t-J}$ in the one hole case. The corresponding real-space 
form of (17) may be rewritten as

$$
G_{1 \sigma}^{\prime \prime}(j, i ; E)=-\pi \sum_{k} e^{-i k \cdot\left(x_{j}-x_{i}\right)} Z_{k}(E) \rho(E),
$$

where $\rho(E)=\sum_{M} \delta\left(E-E_{M}\right)$ is the density of states, and here $Z_{k}$ is understood as being averaged over $M$ at the same energy $E$. The spectral weight $Z_{k}(E)$ describes the overlap of the bare-hole state $c_{k \sigma}\left|\psi_{0}\right\rangle$ with real eigenstates at energy $E$. One may define $E_{k}$ as the lower-bound energy for nonzero $Z_{k}(E)$ at each momentum $k$. In particular, if the overlap of the bare-hole state with the ground state is finite, $E_{k}$ reaches its minimum at the ground-state energy $E_{G}$ at $k_{0}$ determined by $E_{k_{0}}=E_{G}$. If low-lying excitations can be classified as quasiparticlelike, one must have a finite spectral weight at the ground state and its vicinity. In this case, $G_{1 \sigma}^{\prime \prime}$ should become extended (without decay) at large scale of $\left|x_{j}-x_{i}\right|$ when $E=E_{G}$, if only discrete $k_{0}$ 's are involved at $E_{G}$ (a continuum of $k_{0}$ 's would mean a dispersionless of the quasiparticle spectrum which implies an infinite effective mass and localization of the hole). Therefore, for a mobile quasiparticle case, $G_{1 \sigma}^{\prime \prime}$ should become sufficiently extended at large $\left|x_{i}-x_{j}\right|$ if $E$ is close enough to $E_{G}$ [8].

The nonperturbative nature of the present problem is clearly shown in the propagator (13) where each path is weighted by the phase string. The larger the distance is, the longer the phase string is, and the latter cannot be simply broken up into some disconnected "diagrams." The conclusion that $Z=0$ at $E=E_{G}$ means that there is no overlap between the bare hole state $c_{i \sigma}\left|\psi_{0}\right\rangle$ and the true ground state. The doped hole will have to induce a global adjustment of the spin background in order to reach the ground state. In other words, by starting from $c_{i \sigma}\left|\psi_{0}\right\rangle$, one cannot get access to the ground state perturbatively as there is no zeroth-order overlapping. It is also noted that conditions (3) and (5) are crucially responsible for the nonrepairable phase-string effect in (13). They are related to the intrinsic competitive effect of the $t$ - $J$ model. On the other hand, the property of $\left|\psi_{0}\right\rangle$ as the ground state of the undoped antiferromagnet actually does not play a crucial role here. Hence, the whole argument about $Z\left(E_{G}\right)=0$ should still remain robust at least in the weakly doped case. At finite doping, an additional phase due to the fermionic statistics among holes will appear in the matrix (5), but such a sign problem should not invalidate the afore-discussed phasestring effect at least in the small doping case. Thus, in order to correctly understand the long-wavelength, lowenergy physics of the $t$ - $J$ model, a non-perturbativeminded approach should become necessary. Of course, $Z\left(E_{G}\right)=0$ itself does not tell how the nonperturbative approach should be pursued. One has to go back to the original source, i.e., the phase-string effect induced by the hopping, which causes $Z\left(E_{G}\right)=0$. In the one- dimensional case, the key role of the phase string in determining the correct Luttinger-liquid behaviors has already been shown [7]. In the 2D finite-doping case, the phase string effect implies a nonlocal exotic interaction between the charge and spin degrees of freedom. We shall explore the consequences of this effect elsewhere.

In conclusion, we have demonstrated the existence of a singular phase-string effect induced by hopping in the one hole case for a general dimensionality. Such a string phase picked up by the hole from the spin background is shown to be nonrepairable by the low-lying spin dynamics, and thus its quantum interference at long distance dramatically changes the single hole's behavior. We have found that the phase string generally leads to a vanishing spectral weight: i.e., $Z\left(E_{G}\right)=0$, implying a nonperturbative nature of the doped antiferromagnet. We also point out that the phase string is an intrinsic property of the $t-J$ model which should be present as well at finite doping.

The authors acknowledge helpful discussions with D. Frenkel, B. Friedman, T. K. Lee, Y. S. Wu, O. Starykh, G. Baskaran, N. P. Ong, B. Marston, and C. S. Ting. The present work is supported partially by Texas Advanced Research Program under Grant No. 3652182, and by Texas Center for Superconductivity at University of Houston.

*Permanent address: Department of Physics, University of Science and Technology of China, Hefei, Anhui 230026, China.

[1] S. Schmitt-Rink et al., Phys. Rev. Lett. 60, 2793 (1988); C.L. Kane et al., Phys. Rev. B 39, 6880 (1989); B. Shraiman and E. Siggia, Phys. Rev. Lett. 61, 467 (1988); Phys. Rev. B 42, 2485 (1990); Z. Liu and E. Manousakis, Phys. Rev. B 44, 2414 (1991); 45, 2425 (1992); 51, 3156 (1995); E. Louis et al., Phys. Rev. B 48, 9581 (1993); A. Rams̆ak and P. Horsch, Phys. Rev. B 48, 10559 (1993); G. Reiter, Phys. Rev. B 49, 1536 (1994).

[2] E. Dagotto et al., Phys. Rev. B 41, 9049 (1990); M. Boninsegni and E. Manousakis, Phys. Rev. B 45, 4877 (1992); S. Sorella, Phys. Rev. B 46, 11670 (1992); D. Poilblanc et al., Phys. Rev. B 47, 3273 (1993); D.W. Leung and R. J. Gooding, Phys. Rev. B 52, 15711 (1995).

[3] For a review, see E. Dagotto, Rev. Mod. Phys. 66, 763 (1994), and references therein.

[4] P. W. Anderson, Phys. Rev. Lett. 64, 1839 (1990).

[5] W. Marshall, Proc. R. Soc. London A 232, 48 (1955).

[6] H. J. Schulz (private communication).

[7] Z. Y. Weng, Phys. Rev. B 50, 13837 (1994), and references therein.

[8] Here one should be cautious about some special cases where $\rho\left(E_{G}\right)=0$. For example, it occurs at a dimensionality higher than $2 \mathrm{D}$ for a parabolic spectrum, and in $2 \mathrm{D}$ for a massless Dirac spectrum $E_{k}$. 